\title{
Proceso de enfermería aplicado a un paciente con SARS-CoV-2 y Diabetes Mellitus 2
}

\author{
Jahir Francisco Rolando Villarreal de la Cruz'; Milton Carlos Guevara Valtier²; \\ María Magdalena Ramírez Hernández ${ }^{3}$; María de los Ángeles Paz Morales ${ }^{4}$; Martha Pérez Fonseca ${ }^{5}$
}

\begin{abstract}
RESUMEN
Introducción: En diciembre de 2019, Wuhan, capital de la provincia de Hubei (China), se convirtió en el epicentro de una serie de casos de neumonía de causa desconocida. El agente biológico identificado es un betacoronavirus de ácido ribonucleico con envoltura que actualmente se denomina coronavirus de tipo 2 causante del síndrome respiratorio agudo severo. Objetivo: Desarrollar un proceso de enfermería aplicado a un paciente con el binomio coronavirus de tipo 2 causante del síndrome respiratorio agudo severo y Diabetes Mellitus 2. Metodología: Estudio de caso clínico descriptivo, se desarrolló el proceso de enfermería, la valoración fue guiada por el enfoque conceptual de Virginia Henderson, se establecieron diagnósticos, resultados e intervenciones. Presentación del caso: Masculino de 44 años de edad con coronavirus de tipo 2 causante del síndrome respiratorio agudo severo y Diabetes Mellitus 2 que ingresó a la Unidad de Cuidados Intensivos Adultos de un Hospital de Tercer Nivel de Atención. Resultados: Se identificaron los diagnósticos "Limpieza ineficaz de las vías aéreas, hipertermia y riesgo de nivel de glucemia inestable". Conclusión: Mediante las intervenciones se alcanzaron los resultados esperados, el paciente egresó de la unidad de cuidados intensivos 18 días posteriores a su internamiento.
\end{abstract}

Palabras clave: Proceso de enfermería; Síndrome respiratorio agudo grave; Infecciones por coronavirus; Diabetes mellitus (DeCS).

1.Universidad Emiliano Zapata.

ORCID-iD: https://orcid.org/0000-0002-5992-8431

2.Universidad Autónoma de Nuevo León, Facultad de Enfermería.

ORCID-iD: https://orcid.org/0000-0001-7291-3931

3.Universidad Emiliano Zapata.

ORCID-iD: https://orcid.org/0000-0001-9978-5941

4.Universidad Autónoma de Nuevo León, Facultad de Enfermería.

ORCID-iD: https://orcid.org/0000-0002-4111-8449

5. Universidad Veracruzana, Campus Minanititlán.

*Autor para correspondencia: carlos valtier7@hotmail.com

Recibido: $29 / 05 / 2020$

Aceptado: 18/12/2020 


\title{
Nursing process applied to a patient with SARS-CoV-2 and diabetes mellitus \\ type 2
}

\begin{abstract}
Introduction: On December 2019, Wuhan, the capital of the province of Hubei (China), became the epicenter of a series of cases of pneumonia whose cause was unknown. The biological agent was identified as a ribonucleic acid betacoronavirus of with coat which is currently named coronavirus 2, the cause of a severe acute respiratory syndrome. Objective: Develop a nursing process applied to a patient with the binomial comprised by coronavirus 2 which caused him severe acute respiratory syndrome, and diabetes mellitus type 2. Methodology: Descriptive clinical case study, the nursing process was developed, the assessment was guided by the Virginia Henderson conceptual approach; diagnosis, results, and interventions were established. Presentation of the case: 44 years old male with coronavirus 2 , which causes the severe acute respiratory syndrome, and diabetes mellitus type 2, who was admitted in the Intensive Care Unit for Adults in a tertiary care hospital. Results: The diagnosis was "Inefficient cleaning in the airways, hyperthermia, and risk of instable glycemia level". Conclusion: Through the interventions expected results were reached; the patient was dismissed from the intensive care unit 18 days after he was admitted.
\end{abstract}

Key words: Nursing process; Severe Acute Respiratory Syndrome; infections due to coronavirus; diabetes mellitus (DeCS). 


\title{
Processo de enfermagem aplicado a um paciente com SARS-CoV-2 e diabetes mellitus tipo 2
}

\begin{abstract}
ABSTRATO
Introdução: Em dezembro de 2019, Wuhan, capital da província de Hubei (China), tornou-se o epicentro de uma série de casos de pneumonia de causa desconhecida. $O$ agente biológico foi identificado como um betacoronavírus de ácido ribonucléico com revestimento, atualmente denominado coronavírus 2 , causador de síndrome respiratória aguda grave. Objetivo: Elaborar um processo de enfermagem aplicado a um paciente portador do binômio composto pelo coronavírus 2, que lhe causou a síndrome respiratória aguda grave, e diabetes mellitus tipo 2. Metodologia: Estudo de caso clínico descritivo, o processo de enfermagem foi desenvolvido, a avaliação foi norteada pela abordagem conceitual de Virginia Henderson; diagnóstico, resultados e intervenções foram estabelecidos. Apresentação do caso: Homem de 44 anos com coronavírus 2, causador da síndrome respiratória aguda grave, e diabetes mellitus tipo 2, internado em Unidade de Terapia Intensiva para Adultos de um hospital terciário. Resultados: O diagnóstico foi "Limpeza ineficaz das vias aéreas, hipertermia e risco de glicemia instável". Conclusão: Por meio das intervenções foram alcançados os resultados esperados; o paciente teve alta da unidade de terapia intensiva 18 dias após sua internação.
\end{abstract}

Palavras-chave: Processo de enfermagem; Síndrome Respiratória Aguda Grave; infecções por coronavírus; diabetes mellitus (DeCS). 


\section{INTRODUCCIÓN}

En diciembre de 2019, Wuhan, capital de la provincia de Hubei (China), se convirtió en el epicentro de una serie de casos de neumonía de causa desconocida (1). El agente biológico identificado es un betacoronavirus de ácido ribonucleico con envoltura denominado coronavirus 2 del síndrome respiratorio agudo severo (SARS-CoV-2), posee una semejanza filogenética con el coronavirus del SARS (SARSCoV) ${ }^{(1)}$.

Estructuralmente, el SARS-CoV tiene una composición bien definida que comprende 14 residuos de unión que interactúan directamente con la enzima convertidora de angiotensina 2 (ECA2). De estos aminoácidos, 8 se han conservado en el SARS-CoV-2. Aunque se desconocen los mecanismos fisiopatológicos exactos subyacentes a la aparición de SARSCoV-2 (debido a ensayos de laboratorio pendientes), las similitudes genómicas con el SARS-CoV podrían ayudar a explicarlo. Sin embargo, hasta que se inicien estos ensayos de laboratorio, el mecanismo preciso del SARSCoV-2 sigue siendo hipotético (2).

Los síntomas de SARS-CoV-2 aparecen después de un período de incubación de aproximadamente 5,2 días ${ }^{(3)}$. Los síntomas más comunes al inicio de la enfermedad del SARSCoV-2 son fiebre, tos y fatiga, mientras que otros síntomas incluyen producción de esputo, dolor de cabeza, hemoptisis, diarrea, disnea y linfopenia, rinorrea, estornudos y dolor de garganta. Algunos de los casos muestran un infiltrado en el lóbulo superior del pulmón que se asocia con una disnea creciente con hipoxemia ${ }^{(3)}$. El diagnóstico se realiza mediante reacción en cadena de la polimerasa con transcriptasa inversa (RT-PCR) a través de la identificación de ácidos nucleicos del SARSCoV-2 o por medio de la secuenciación del gen viral. También es posible con muestras de exudado faríngeo/nasofaríngeo, esputo, heces o sangre. Otro método es el aislamiento del virus de cultivos de células epiteliales respiratorias ${ }^{(4)}$.

Las principales formas de transmisión del SARS-CoV-2, son a través de gotas y contacto. La transmisión por gotas $>$ 5-10 $\mu \mathrm{m}$ de diámetro ocurre en forma directa entre una persona infectada y un contacto cercano generalmente dentro del diámetro de 1 a 2 metros de distancia y el virus ingresa por las mucosas nasal, oral y ocular. Las gotas también pueden contaminar superficies y objetos cercanos a la persona infectada y transmitirse indirectamente a través de la contaminación de las manos que posteriormente pueden estar en contacto con las mucosas. Esta vía de contagio es muy importante dado que el virus demuestra una viabilidad de 3 o más días en superficies como el plástico, el metal u otros materiales. Asimismo, puede transmitirse en períodos presintomáticos y desde personas asintomáticas. La transmisión a través del aire es menos frecuente $y$ requiere que el virus permanezca contenido en partículas $<5 \mu \mathrm{m}$ de diámetro que pueden aerosolizarse $y$ mantenerse durante prolongados intervalos de tiempo en el aire y transmitirse a longitudes superiores de 1 a 2 metros, lo anterior es probable en condiciones y entornos específicos en los que se llevan a cabo procedimientos que producen aerosoles como lo son la intubación/extubación endotraqueal, broncoscopía, toma de muestras del sistema respiratorio, reanimación cardiopulmonar; entre otros más ${ }^{(5)}$.

En la actualidad, se han diseñado vacunas que promueven resultados eficaces $y$ alentadores para SARS-CoV-2; sin embargo, aún existe consenso para la promoción de terapias y protocolos específicos que sean aprobados por la Administración de Alimentos y Medicamentos de los Estados Unidos (FDA) ${ }^{(2)}$. 
En el contexto mexicano se ha iniciado con la fase 1 para la aplicación de la vacuna (personal de salud en primera línea), otras vacunas continúan en ensayos clínicos y protocolos de uso compasivo con base en la actividad in vitro y con una experiencia clínica limitada; incluso la efectividad de tratamientos farmacológicos para el tratamiento de pacientes con esta enfermedad continúa en prueba ${ }^{(6)}$.

Con la finalidad del estudio de los factores de riesgo existe evidencia de que el SARS-CoV-2, así como otros tipos de coronavirus patógenos en los seres humanos tienen capacidad de introducirse en las células pulmonares acoplándose a la ECA2 (7). La expresión del receptor de la ECA2 aumenta en sujetos con enfermedades crónicas como Diabetes Mellitus (DM) tipo 1 y 2 (DM-I y DMII) (8). Estudios recientes refieren que la DM tratada con drogas que estimulan la expresión de la ECA2, aumentan el riesgo de desarrollar la enfermedad por SARS-CoV-2 en su forma más severa y fatal ${ }^{(9)}$.

En México el 27 de febrero de 2020 se hizo público el caso de un hombre con resultado positivo para la prueba de SARS-CoV-

2. La persona se presentó en el Instituto Nacional de Enfermedades Respiratorias (INER) para consulta, con el historial de haber visitado Bérgamo, Italia, en tal lugar estuvo en contacto con un sujeto contagiado. Al día siguiente, se confirmó que este era el primer caso de SARSCoV-2 en México por el Instituto de Diagnóstico y Referencia Epidemiológica (INDRE) ${ }^{(10)}$.

El 30 de enero de 2020, la Organización Mundial de la Salud (OMS) informó sobre la emergencia de salud pública mundial que representaba la aparición repentina del SARSCoV-2, posteriormente, el 11 de marzo de 2020 la OMS calificó al SARS-CoV-2 como una pandemia (11). En este sentido enfermería siempre ha jugado un papel importante en la prevención y control de infecciones, acciones de aislamiento para la protección de la salud de personas inmunocomprometidas y además juega un rol de amplia importancia por su impacto en la salud pública ${ }^{(12)}$ por lo que ante la situación de una pandemia enmarcada como un problema global se requiere de la participación de la fuerza laboral de este grupo de profesionales. Es así como este gremio se encuentra involucrado demostrando responsabilidad y sentido de servicio ante la respuesta al SARS-CoV-2 y continuarán en la primera línea de atención al paciente ${ }^{(13)}$.

\section{Objetivo}

El objetivo del presente estudio fue desarrollar un proceso de enfermería aplicado a un paciente con el binomio coronavirus 2 del síndrome respiratorio agudo severo y Diabetes Mellitus 2 (DM-II).

\section{METODOLOGÍA}

Se desarrolló un estudio de caso clínico descriptivo en la UCIA de un hospital ubicado en el noreste de México durante el mes de abril del 2020 a un paciente con SARS-CoV-2 y DMII. Se estructuró de acuerdo con las cinco etapas del PE y con la utilización del enfoque filosófico conceptual de Virginia Henderson, que otorga al profesional de enfermería un rol complementario y suplementario en la satisfacción de las 14 necesidades básicas del ser humano con el objetivo de lograr la independencia de la persona considerando como base las características individuales de quien requiere la intervención de enfermería ${ }^{(14)}$. La selección de este marco conceptual filosófico recae en que su principal foco de atención se enfoca a la valoración en el marco del PE (14). Aunado a lo anterior para facilitar la ejecución de la valoración y precisar las áreas a evaluar se consideró la organización y jerarquización de las necesidades humanas propuestas por 
Maslow y retomadas por Henderson, por lo tanto, este marco conceptual filosófico se consideró adecuado para su aplicación en este PE (15).

Se valoraron las 14 necesidades básicas; sin embargo, sólo se abordaron las de mayor compromiso en cuanto a gravedad expresadas por el paciente en estudio, las cuales fueron "oxigenación, mantener la temperatura del cuerpo en los rangos normales y seguridad". Se establecieron diagnósticos, intervenciones y resultados basados en la North American Nursing Diagnosis Association (NANDA), la clasificación de intervenciones de enfermería (NIC) y la de resultados de enfermería (NOC) ${ }^{(15-}$ 17).

\section{PRESENTACIÓN DEL CASO}

\section{Antecedentes personales}

Paciente masculino de 44 años, residente de Monterrey, Nuevo León, México, soltero, católico, con DM-II desde hace un año, sin tratamiento, amputación del dedo meñique del pie derecho.

\section{Valoración}

Ingresa el 12 de abril del 2020 al servicio de admisión, ingresa consciente y orientado en espacio, tiempo y persona, con biotipo medio líneo y constitución mesomórfica. Al ingreso presenta tos con expectoración con una evolución de 7 días, acude al servicio de salud por presentar disnea e hipoxia moderada $(90 \%$ O2), con alteración en signos vitales Temperatura: $38.5^{\circ} \mathrm{C}$ (hipertermia), Presión arterial: 99/54 mm/Hg, Frecuencia cardiaca: 79x', Frecuencia Respiratoria (FR): 30x' (taquipnea), Saturación de oxígeno: $84 \%$ (hipoxia moderada), Peso: $70 \mathrm{Kg}$, Talla: 1.71 metros, Índice de masa corporal (IMC): 23.94. Se realiza la prueba de RT-PCR para SARS-CoV-2 mediante hisopado nasofaríngeo, obteniendo resultado positivo, motivos por los que se decidió su ingreso a hospitalización, posterior a esta valoración se decide realizar traslado directo a la UCIA donde se realiza el procedimiento invasivo. Se procede a inducir estado de sedación, orointubado con apoyo ventilatorio en modo asisto-control (AC) ciclado por volumen, con parámetros de FR 26x', PEEP 10, $\mathrm{FiO} 250 \%$, VT $420 \mathrm{ml}$. Presenta abundantes secreciones mucopurulentas; sonda orogástrica y fórmula para alimentación enteral diseñada para paciente diabético a $20 \mathrm{ml} /$ hora; con catéter venoso central de tres lúmenes en vena subclavia derecha, permeable y con ministración de Propofol a $50 \mu \mathrm{g} / \mathrm{Kg} / \mathrm{min}$, infusión de Dexmedetomidina a $1 \mu \mathrm{g} / \mathrm{Kg} / \mathrm{min}$, norepinefrina a $3 \mu \mathrm{g} / \mathrm{Kg} / \mathrm{min}$, insulina rápida humana a 4 unidades/hora, plan de líquidos a base de solución $\mathrm{NaCl}$ al $0.9 \%$ a $41 \mathrm{ml} /$ hora, se monitorizó la presión arterial vía invasiva en arteria radial de miembro superior derecho, peristalsis audible, sonda transuretral a derivación con diuresis color amarillo oro a 0.5 $\mathrm{ml} / \mathrm{Kg} / \mathrm{hora}$.

\section{Exámenes auxiliares}

RT-PCR SARS-CoV-2: Positivo $121.07 \mathrm{mg} / \mathrm{L}$. Biometría hemática: Leucocitosis $20.10 \mathrm{~K} / \mathrm{uL}$, neutropenia $18.0 \mathrm{~K} / \mathrm{uL}$, eosinopenia $0.00 \mathrm{~K} / \mathrm{uL}$, linfopenia $0.58 \mathrm{~K} / \mathrm{uL}$. Química sanguínea: glucosa en sangre $207 \mathrm{mg} / \mathrm{dL}$. Tiempos de coagulación: tiempo de protrombina $12.9 \mathrm{seg}$, tiempo de tromboplastina $31.7 \mathrm{seg}$. Gasometría arterial: pH 7.46, pCO2 $29 \mathrm{~mm} / \mathrm{Hg}, \mathrm{pO} 267$ $\mathrm{mm} / \mathrm{Hg}, \mathrm{HCO} 220.3 \mathrm{mmol} / \mathrm{L}, \mathrm{Na}+149 \mathrm{mmol} / \mathrm{L}$, $\mathrm{K}+3.6 \mathrm{mmol} / \mathrm{L}$, Lactato $1.0 \mathrm{mmol} / \mathrm{L}$. Estudios de gabinete: Radiografía con evidencia de infiltrados bronquio alveolares bilaterales y opacidad en vidrio esmerilado. 


\section{Principales evidencias de la valoración por necesidades}

1. Necesidad de oxigenación: Disnea, vía aérea artificial (orointubación), frecuencia respiratoria (FR: 26x'), retención de las secreciones (abundantes mucopurulentas), hipoxemia moderada (Saturación de oxígeno: 90\%).

2. Necesidad de mantener la temperatura del cuerpo en los rangos normales: temperatura: $38.5^{\circ}$ C, Leucocitos $20.10 \mathrm{~K} / \mathrm{uL}$, frecuencia cardiaca: 79x', presión arterial: 99/54 mm/Hg.

3. Necesidad de seguridad: Sexo masculino, edad 44 años, DM-II desde hace un año y seis meses, amputación de dedo meñique del pie derecho, glucosa en sangre de $207 \mathrm{~mm} / \mathrm{Hg}$.

Posterior al destete de sedación y vasopresor, el paciente se mantiene consciente. Orointubado referido a quirófano, regresa con cánula de traqueostomía con oxígeno al 21\% mantiene saturación de oxígeno hasta $94 \%$, secreciones escasas hialinas. Continúa aspiración de secreciones con sistema cerrado. Después de la administración de terapia farmacológica y no farmacológica, el paciente se mantiene afebril hasta su egreso. Metabólicamente, posterior al destete de la infusión de insulina rápida humana, el paciente se mantiene euglucémico. Mientras, se prepara el plan de alta y el nuevo tratamiento para el manejo de la DM-II.

\section{Plan de cuidado de enfermería}

Ver tablas de la 1 a la 3.

Tabla 1. Plan de Cuidados de Enfermería 1. Necesidad alterada: oxigenación

Diagnóstico: (00031) Limpieza ineficaz de las vías aéreas, relacionado con cuerpo extraño en la vía aérea (orointubación), retención de las secreciones, manifestado por la alteración de la frecuencia respiratoria (FR: 26x'), cantidad excesiva de esputo (abundantes mucopurulentas).

\begin{tabular}{|c|c|}
\hline Resultados & $\begin{array}{l}\text { Intervenciones de enfermería, Tipo de } \\
\text { intervención y Nivel de relación }\end{array}$ \\
\hline $\begin{array}{l}\text { Resultado seleccionado: } \\
\text { (0410) Estado respiratorio: permeabilidad } \\
\text { de las vías aéreas } \\
\text { Indicador seleccionado: } \\
\text { (41012) Capacidad de eliminar secreciones } \\
\text { Escala de Medición: } 1 \text { a } 5\left(^{*}\right) \\
\text { Puntuación Diana: Mantener: } 3 \text {, Aumentar: } \\
4 \\
\text { (41020) Acumulación de esputos } \\
\text { Escala de Medición: } 1 \text { a } 5 \text { (+) } \\
\text { Puntuación Diana: Mantener: } 3 \text {, Aumentar: } \\
4 \\
\text { Indicadores que no aplican: } \\
\text { (41002) Ansiedad, (41003) Asfixia, (41004) } \\
\text { Frecuencia respiratoria, (41005) Ritmo } \\
\text { respiratorio, (41007) Ruidos respiratorios } \\
\text { patológicos, (41011) Temor, (41013) } \\
\text { Aleteo nasal, (41014) Jadeo, (41015) } \\
\text { Disnea en reposo, (41016) Disnea de }\end{array}$ & $\begin{array}{l}\text { (3160) Aspiración de las vías aéreas } \\
\text { Usar precauciones universales e incluir equipo de } \\
\text { protección personal } \\
\text { Monitorizar el estado de oxigenación del paciente } \\
\text { (niveles de saturación de oxígeno) } \\
\text { Determinar la necesidad de aspiración } \\
\text { Utilizar aspiración de sistema cerrado } \\
\text { Realizar aspiración orotraqueal, según corresponda } \\
\text { Controlar y observar el color, cantidad y consistencia } \\
\text { de las secreciones } \\
\text { (0840) Cambio de posición } \\
\text { Colocar sobre un colchón terapéutico adecuado } \\
\text { Vigilar el estado de oxigenación antes y después del } \\
\text { cambio de posición, según corresponda } \\
\text { Colocar en la posición terapéutica específica (decúbito } \\
\text { prono) } \\
\text { Colocar en posición de alineación corporal correcta } \\
\text { Poner apoyos en las zonas edematosas. } \\
\text { Tipo de intervención: Interdependiente, } \\
\text { Nivel de relación: Suplencia }\end{array}$ \\
\hline
\end{tabular}


esfuerzo leve, (41017) Profundidad de la inspiración, (41018) Uso de músculos accesorios, (41019) Tos, (41021)

Respiraciones agónicas

\section{Notas:}

(*) 1 = Desviación grave del rango normal, 2 = Desviación sustancial del rango normal, 3 = Desviación moderada del rango normal, $4=$ Desviación leve del rango normal, $5=$ Sin desviación del rango normal.

(+) 1 = Grave, 2 = Sustancial, $3=$ Moderado, $4=$ Leve, $5=$ Ninguno.
Nota de evolución: Orointubado referido a quirófano donde se instala cánula de traqueostomía con oxígeno al 21\%, mantiene saturación de oxígeno hasta $94 \%$, secreciones escasas hialinas.

Fuente: NANDA (16), NIC (17), NOC (18).

Tabla 2. Plan de Cuidados de Enfermería 2. Necesidad alterada: mantener la temperatura del cuerpo en los rangos normales.

Diagnóstico: (00007) Hipertermia, relacionado con enfermedad, manifestado por la temperatura: $38.5^{\circ} \mathrm{C}$.

\begin{tabular}{|c|c|}
\hline Resultados & $\begin{array}{c}\text { Intervenciones de enfermería, Tipo de intervención } \\
\text { y Nivel de relación }\end{array}$ \\
\hline $\begin{array}{l}\text { Resultado seleccionado: } \\
\text { (0800) Termorregulación } \\
\text { Indicador seleccionado: } \\
\text { (80019) Hipertermia } \\
\text { Escala de medición: } 1 \text { a } 5\left(^{*}\right) \\
\text { Puntuación Diana: Mantener: 4, Aumentar: } \\
5 \\
\text { Indicadores que no aplican: } \\
\text { (80001) Temperatura cutánea aumentada, } \\
\text { (80003) Cefalea, (80004) Dolor muscular, } \\
\text { (80005) Irritabilidad, (80006) Somnolencia, } \\
\text { (80007) Cambios de coloración cutánea, } \\
\text { (80008) Contractura muscular, (80009) } \\
\text { Presencia de piel de gallina cuando hace } \\
\text { frío, (80010) Sudoración con el calor, } \\
\text { (80011) Tiritona con el frío, (80012) } \\
\text { Frecuencia del pulso radial, (80013) } \\
\text { Frecuencia respiratoria, (80014) } \\
\text { Deshidratación, (80015) Comodidad } \\
\text { térmica referida, (80017) Frecuencia } \\
\text { cardíaca apical, (80018) Disminución de la } \\
\text { temperatura cutánea, (80020) Hipotermia, } \\
\text { (80021) Calambres por calor, (80022) }\end{array}$ & $\begin{array}{l}\text { (3900) Regulación de la temperatura } \\
\text { Usar precauciones universales } \\
\text { Usar el equipo de protección personal que sea } \\
\text { adecuado } \\
\text { Instaurar un dispositivo de monitorización de } \\
\text { temperatura central continua } \\
\text { Utilizar un colchón de enfriamiento, mantas de agua } \\
\text { circulante, compresas de hielo } \\
\text { Administrar medicamentos antipiréticos. } \\
\text { Tipo de intervención: Interdependiente, } \\
\text { Nivel de relación: Suplencia } \\
\text { Nota de evolución: Se mantiene normotermico. }\end{array}$ \\
\hline
\end{tabular}


Golpe de calor, (80023) Congelación.

Notas:

(*) $1=$ Grave, 2 = Sustancial, $3=$

Moderado, 4 = Leve, 5 = Ninguno.

Fuente: NANDA ${ }^{(16)}$ NIC ${ }^{(17)}$, NOC $^{(18)}$.

Tabla 3. Plan de Cuidados de Enfermería 3. Necesidad alterada: seguridad.

Diagnóstico: (00179) Riesgo de nivel de glucemia inestable, relacionado con DM-II desde hace un año y seis meses, amputación de dedo meñique del pie derecho, control inadecuado de la glucemia (glucosa en sangre de $207 \mathrm{~mm} / \mathrm{Hg}$ ).

\begin{tabular}{|c|c|}
\hline Resultados & $\begin{array}{c}\text { Intervenciones de enfermería, Tipo de intervención } \\
\text { y Nivel de relación }\end{array}$ \\
\hline $\begin{array}{l}\text { Resultado seleccionado: } \\
\text { (2300) Nivel de glucemia } \\
\text { Indicador seleccionado: } \\
\text { (230001) Concentración sanguínea de } \\
\text { glucosa. } \\
\text { Escala de Medición: } 1 \text { a } 5\left(^{*}\right) \\
\text { Puntuación Diana: Mantener: } 4 \text {, Aumentar: } \\
5 \\
\text { Indicadores que no aplican. } \\
(230004) \text { Hemoglobina glucosilada, } \\
(230005) \text { Fructosamina, (230007) Glucosa } \\
\text { en orina, (230008) Cetonas en orina. } \\
\text { Notas: } \\
\text { (*) } 1 \text { = Desviación grave del rango normal, } \\
2=\text { Desviación sustancial del rango } \\
\text { normal, } 3=\text { Desviación moderada del } \\
\text { rango normal, } 4 \text { = Desviación leve del } \\
\text { rango normal, } 5 \text { = Sin desviación del rango } \\
\text { normal. }\end{array}$ & $\begin{array}{l}\text { (2300) Nivel de glucemia } \\
\text { Realizar el lavado de manos } \\
\text { Usar precauciones universales } \\
\text { Usar el equipo de protección personal que sea } \\
\text { adecuado } \\
\text { Vigilar la glucemia en la sangre } \\
\text { Administrar insulina, según prescripción } \\
\text { Proporcionar ayuda en el ajuste de un régimen para } \\
\text { evitar y tratar la hiperglucemia (insulina). } \\
\text { Tipo de intervención: Interdependiente, } \\
\text { Nivel de relación: Suplencia } \\
\text { Nota de evolución: Destete de la infusión de insulina } \\
\text { rápida humana, se mantiene euglucémico. }\end{array}$ \\
\hline
\end{tabular}

Fuente: NANDA ${ }^{(16)}$, NIC ${ }^{(17)}$, NOC (18).

\section{DISCUSIÓN}

En sus escritos, Virginia Henderson considera que las personas poseen capacidades y recursos reales y potenciales para alcanzar su independencia y satisfacer sus necesidades básicas, con el propósito de preservar en estado óptimo la propia salud ${ }^{(19)}$. En ocasiones estas capacidades y recursos merman, se reducen de forma completa o incompleta, temporal o permanentemente. Es entonces cuando aparece una dependencia, como en el caso previamente expuesto.

La particular neumonía del SARS-CoV-2 acontece en mayor medida como una inflamación del tejido alveolar, que produce tos seca y aparentemente de consolidación no 
exudativa. No obstante, hasta un $34 \%$ de los pacientes con SARS-CoV-2 manifestaron tos productiva, en los cuales las técnicas de eliminación de secreciones resultan favorables. Las técnicas de eliminación de secreciones estarían destinadas a pacientes con exudado bronquial, y únicamente cuando se dificulte la expulsión de las secreciones por cuenta propia (20), como en el caso de los pacientes con orointubación o cánula de traqueostomía. Resultó de vital importancia vigilar los signos vitales, especialmente la saturación de oxígeno mediante pulsioximetría (SpO2), con el propósito de salvaguardar al paciente. Prioritariamente de forma remota por medio de monitores a distancia ${ }^{(20)}$.

La evidencia acerca del uso de la posición prono en pacientes con SARS-CoV-2 es limitada. Sin embargo, la tendencia del SARS-CoV-2 para perjudicar zonas periféricas y dorsales de los pulmones provee las circunstancias óptimas para una respuesta de oxigenación positiva al posicionamiento prono ${ }^{(11)}$. El decúbito prono sugerido por Barrantes y Vargas ${ }^{(21)}$ reordena las presiones pleurales volviéndose más uniformes, así como que el gradiente de presión sea cercano a cero, de modo que el volumen pulmonar se distribuya de manera análoga. Además, produce un significativo reclutamiento alveolar sobre áreas dorsales, en consecuencia, la oxigenación presenta mejoría, hecho que coincide con lo que ocurrió en este caso clínico y el propuesto por Vásquez (22). La responsabilidad de estos cambios posturales recae sobre el equipo de enfermería que debe prepararse para minimizar los riesgos y conocer las implicaciones asociadas al procedimiento (21).

La hipertermia se encuentra presente en el $43,8 \%$ de los pacientes al momento de la admisión y es el síntoma más común, presente entre el 64,7 y el $98 \%$ de los pacientes internados ${ }^{(1,2,23)}$. Los pacientes con hipertermia deben ser controlados activamente. El tratamiento de primera línea para las fiebres incluye terapia antipirética como paracetamol (24). De acuerdo con el PE de Montero (25), la administración de antipiréticos tiene un efecto positivo en la evolución del paciente sobre el control de la temperatura corporal, situación que coincide con este caso.

La epidemia mundial del SARS-CoV-2 tiene implicaciones inmediatas para la terapia de trastornos metabólicos comunes como la DMII. La insulina es la terapia de reducción de glucosa de elección para la enfermedad aguda relacionada con el coronavirus en el hospital, pues se ha utilizado ampliamente durante décadas para controlar la glucosa en sujetos hospitalizados en estado crítico con DM-II. Entre los agentes disponibles para el tratamiento de enfermedades agudas complicadas por DM-II, la insulina ha sido el agente más utilizado en seres humanos con infecciones bacterianas o virales y en pacientes hospitalizados en estado crítico. Sin embargo, existe poca información sobre los posibles beneficios o riesgos de la insulina en el contexto de la infección aguda por coronavirus (26).

En cuanto a casos de que aborden SARS-CoV-2 los reportes son escasos, pero concluyen que los cuidados de enfermería proporcionados en este PE en conjunto con los protocolos a seguir con un paciente con este diagnóstico respiratorio ${ }^{(27)}$ cubren las esferas de atención para la evolución favorable del paciente con SARS-CoV-2.

Se recomienda considerar este caso clínico como una propuesta que sirva como referente para la práctica de atención de enfermería al brindar cuidados a personas con SARS-CoV-2 y DM-II con la finalidad de lograr resultados que orienten a una práctica de calidad y basada en la seguridad del paciente bajo el sistema de valoración de Henderson para dar un soporte filosófico de enfermería a la valoración física, 
esto permitirá a los profesionales evaluar paulatinamente el nivel de dependencia de los pacientes que experimenten esta situación clínica.

\section{CONCLUSIONES}

Los resultados planteados mejoraron considerando las intervenciones con base en los diagnósticos establecidos, en el caso del diagnóstico 1 los resultados partieron de 1 (desviación grave del rango normal) y 1 (grave) a 4 (desviación leve del rango normal) y 4 (leve), respectivamente, en el diagnóstico 2 los resultados se comportaron de la siguiente manera: 1 (grave) a 5 (ninguno), mientras que en el diagnóstico 3 los resultados fueron desde 1 (desviación grave del rango normal) a 5 (sin desviación grave del rango normal). Se lograron los resultados esperados para el egreso del paciente de la UCIA 18 días posteriores a su internamiento con un grado de dependencia moderado. Se concluye que el PE presentado es eficaz para el tratamiento de los pacientes con SARS-CoV-2 y con patología de base DM-II.

\section{CONFLICTO DE INTERESES}

Ninguno.

\section{FINANCIAMIENTO}

No existió.

\section{REFERENCIAS BIBLIOGRÁFICAS}

1. Guan W, Ni Z, Hu Y, Liang W, Ou C, He J, et al. Clinical Characteristics of Coronavirus Disease 2019 in China. NEJM Catal [Internet]. 2020 [consultado Mayo 2020]; 382(18): 1708-1720. Disponible
https://www.nejm.org/doi/full/10.1056/NEJMo a2002032

2. Huang C, Wang Y, Li X, Ren L, Zhao J, Hu Y, et al. Clinical features of patients infected with 2019 novel coronavirus in Wuhan, China. Lancet [Internet]. 2020 [consultado Mayo 2020]; 395(10223): 497-506. Disponible en: https://doi.org/10.1016/S0140-6736(20)301835

3. Rothan HA, Byrareddy, SN. The epidemiology and pathogenesis of coronavirus disease (COVID-19) outbreak. J Autoimmun [Internet]. 2020 [consultado Mayo 2020]; 109(102433): 1$4 . \quad$ Disponible en: https://doi.org/10.1016/j.jaut.2020.102433

4. Calvo C, García M, Carlos JC, Vázquez JL. Recommendations on the clinical management of the COVID-19 infection by the «new coronavirus» SARS-CoV-2. An Pediatr (Bar) [Internet]. 2020 [consultado Mayo 2020]; 92(4): 241.e1-241.e11. Disponible en: https://doi.org/10.1016/j.anpede.2020.02.002

5. Herrera F. Nuevo coronavirus SARS-COV-2 y enfermedad COVID-19 La pandemia que cambió al mundo. Hematología [Internet]. 2020 [consultado Mayo 2020]; 24(Extraordinario): 4$12 . \quad$ Disponible en: http://revistahematologia.com.ar/index.php/Re vista/article/view/271/289

6. Smith T, Bushek J, LeClaire A, Prosser T. COVID-19 Drug Therapy. [Internet]. Elsevier. 2020 [actualizado Mayo 2020; consultado Mayo 2020]. Disponible en: https://www.elsevier.com/_data/assets/pdf_file /0007/988648/COVID-19-Drug-Therapy_20205-14.pdf

7. Lamas J, Alonso M, Fernández J, Saavedra, J. Supresión de angiotensina II en la infección por el virus SARS-CoV-2: una propuesta terapéutica. Nefrología [Internet]. 2020 [consultado Mayo 2020]. Disponible en: https://doi.org/10.1016/j.nefro.2020.04.006 
8. Soler MJ, Lloveras J, Batle D. Enzima conversiva de la angiotensiva 2 y su papel emergente en la regulación del sistema reninaangiotensina. Med Clin (Barc) [Internet]. 2007 [consultado Mayo 2020]; 131(6):230-236. Disponible

en: https://doi.org/10.1157/13124619

9. Fang L, Karakiulakis G, Roth M. Are patients with hypertension and Diabetes Mellitus at increased risk for COVID-19 infection? Lancet Respir Med [Internet]. 2020 [consultado Mayo 2020]; 8(4): e21. Disponible en: https://doi.org/10.1016/S2213-2600(20)301168

10. Aragón R, Vargas I, Miranda MG. COVID-19 por SARS-CoV-2: la nueva emergencia de salud. Rev Mex Pediatr [Internet]. 2019 [consultado Mayo 2020]; 86(6): 213-218. Disponible en: https://dx.doi.org/10.35366/91871

11. Ríos F, Risso-Vásquez A, Díaz-Ballve, L. Enfermedad por coronavirus 2019 (COVID-19). Aspectos de interés para cuidados críticos. Revisión narrativa. Rev Arg de Ter Int [Internet]. 2020 [consultado Mayo 2020]: 1-12. Disponible en:

http://revista.sati.org.ar/index.php/Ml/article/vi ew/698

12. Mo Y, Deng L, Zhang L, Lang Q, Liao C, Wang $\mathrm{N}$, et al. Work stress among Chinese nurses to support Wuhan for fighting against the COVID19 epidemic. J Nurs Manag [Internet]. 2020 [consultado Mayo 2020]. Disponible en: https://doi.org/10.1111/jonm.13014

13. Choi KR, Skrine JK, Logsdon MC. Nursing and the novel coronavirus: Risks and responsibilities in a global outbreak. J Adv Nurs [Internet]. 2020 [consultado Mayo 2020]: 1-2. Disponible en: https://doi.org/10.1111/jan.14369

14. Barragan T, Gavilanes M, Lombeyda G, Muñoz M. Modelo de Virginia Henderson en la Influenza AH1N1. Talentos [Internet]. 2018 [consultado Mayo 2020]: 2018(1); 704-712. Disponible

en:

https://talentos.ueb.edu.ec/index.php/talentos/ article/view/120
15. Delgado $M$, Hernández $C M$, Ostiguín RM. Fundamentos Filosóficos de la propuesta de Virginia Avenel Henderson. Enfermería universitaria [Internet]. 2020 [consultado Octubre 2020]; 4(1): 24-27. Disponible en: http://revista-

enfermeria.unam.mx/ojs/index.php/enfermeria universitaria/ar

16. Herdman $H$, Kamitsuru S. NANDA. Diagnósticos enfermeros. Definiciones y clasificación 2018-2020. Edición hispanoamericana. 11 ed. España: Elsevier; 2018.

17. Bulecheck $G$, Butcher $H$, Dochterman J, Wagner C. Clasificación De Intervenciones De Enfermería (NIC). 6a ed. España: Elsevier; 2014.

18 Moorhead S, Johnson M, Mass M. Swanson E. Clasificación De Resultados De Enfermería (NOC). $5^{\text {a }}$ ed. España: Editorial Elsevier; 2014.

19. Jiménez $A B$, Salinas MT, Sánchez T. Algunas reflexiones sobre la filosofía de Virginia Henderson. Rev Enferm IMSS [Internet]. 2004 [consultado Mayo 2020]; 12(2); 61-63. Disponible en: https://www.medigraphic.com/pdfs/enfermeria imss/eim-2004/eim042a.pdf

20. Arbillaga $A$, Pardàs $M$, Escudero $R$, Rodríguez $\mathrm{R}$, Alcaraz V, Llanes S, Ríos A, et al. Fisioterapia respiratoria en el manejo del paciente con covid-19: recomendaciones generales. SEPAR [Internet]. 2020 [consultado Mayo 2020]; 1(26). Disponible en: http://svmefr.com/wpcontent/uploads/2020/03/COVID19-SEPAR26_03_20.pdf

21. Morales LFB. Guía de cuidados de enfermería para el decúbito prono en Síndrome de Distress Respiratorio Agudo asociado a COVID-19: Revisión Integrativa. Rev Med Costa [Internet] Rica. 2020 [consultado Mayo 2020]; 85(629); 58-67. Disponible en: http://www.revistamedicacr.com/index.php/rm cr/article/viewFile/293/270 
22. Vásquez JA. Proceso de Atención de Enfermería en paciente de 69 años de edad con Neumonía por COVID-19. [Tesis de Licenciatura]. Ecuador: Universidad Técnica de Babahoyo, Facultad de Ciencias de la Salud; 2020.

23. Wang W, Tang J, Wei F. Updated understanding of the outbreak of 2019 novel coronavirus (2019-nCoV) in Wuhan, China. J Med Virol [Internet]. 2020 [consultado Mayo 2020]; 92(4): 441-447. Disponible en: https://doi.org/10.1002/jmv.25689

24. Sohrabi C, Alsafi Z, O'Neill N, Khan $M$, Kerwan A, Al-Jabir A, et al. World Health Organization declares global emergency: A review of the 2019 novel coronavirus (COVID19). Int J Surg [Internet]. 2020 [consultado Mayo 2020]; 76(2020); 71-76. Disponible en: https://doi.org/10.1016/j.ijsu.2020.02.034

25. Montero JD. Proceso de Atención de Enfermería en paciente de 60 años con Neumonía por COVID. [Tesis de Licenciatura]. Ecuador: Universidad Técnica de Babahoyo, Facultad de Ciencias de la Salud; 2020.

26. Drucker DJ. Coronavirus Infections and Type 2 Diabetes - Shared Pathways with Therapeutic Implications. Endocr Rev [Internet]. 2020 [consultado Mayo 2020]; 41(3): 1-13. Disponible en: https://doi.org/10.1210/endrev/bnaa011

27. Fernández G, Padilla L, Requena MV. Plan de cuidados estandarizados de infección por coronavirus (Covid-19). Enferm Docente [Internet]. 2020 [consultado Octubre 2020]; 112(1): 61-65. Disponible en: http://www.huvv.es/servicioandaluzdesalud/hu $\mathrm{vv/sites/default/files/revistas/Plan \% 20de \% 20C}$ uidados\%20Estandarizados.pdf 